\title{
Skyfall oder mythische Hyleme zum Herabkommen aus dem Himmel
}

\section{Der Befund in ägyptischen literarischen Texten}

\begin{abstract}
This contribution deals with passages from ancient Egyptian literature (narratives, wisdom teachings, speeches and discourses as well as select epics and spells of the Graeco-Roman magical papyri) mentioning subjects and objects descending from sky to earth. Among these are e.g. divinities, demons and heavenly bodies such as stars and comets. Furthermore, the sky itself could fall down on earth. Additionally, an excursus discusses rain and other weather phenomena, prominently the 'rain miracle' during the reign of emperor Marcus Aurelius.
\end{abstract}

\section{Einleitung}

Dieser Beitrag hat zum Ziel, mythische Hyleme zu besprechen, die in ägyptischen literarischen Texten vorkommen ${ }^{1}$. Diese Werke werte ich in einem anderen Projekt hinsichtlich ihrer Aussagen zu Kultpraktiken aus ${ }^{2}$. Es stellt sich heraus, dass narrative, instruktive und diskursive Texte Informationen bieten, die rituelle Texte bestätigen oder sogar ergänzen ${ }^{3}$. Auch für das Studium von Elementen, die vom Himmel kommen, enthalten die literarischen Texte faszinierendes Wissen. Ähnlich wie bei den kurzen mythischen Präzedenzfällen in magischen und divinatorischen Texten konnten Mythen auch Eingang in die Erzählliteratur oder in

1 Zur hier verwendeten Terminologie s. C. Zgoll, „Grundlagen der hylistischen Mythosforschung“, im vorliegenden Band.

2 Habilitationsschrift, Leipzig 2021: Kultpraxis in der altägyptischen Literatur.

3 Es werden in diesem Beitrag keine mythologischen Traktate besprochen, in denen z. B. Götter auf die Erde kommen (bis auf den Text Mythos von der Himmelskuh, s. u.), oder medizinischmagische und divinatorische Texte, in denen der Praktiker Götter und Dämonen evoziert, Götter zu Festen und Orakeln in ihren Kultbildern im Tempel einwohnen lässt oder Heilmittel vom Himmel kommen. Ein Beispiel dafür ist ein Spruch gegen den Bösen Blick, für den die Göttin Hathor vom Himmel kommt (vgl. Fischer-Elfert 2014). 
Weisheitstexte finden - der Definition von Christian Zgoll folgend ${ }^{4}$ : „Ein Mythos ist ein polymorpher und polystrater Erzählstoff, in dem sich transzendierende Auseinandersetzungen mit Erfahrungsgegenständen verdichten.“ Die folgenden Zeilen bieten daher handfeste und dauerhafte Dinge, aber auch Götterboten und Dämonen, die nach ägyptischer Anschauung vom Himmel auf die Erde kamen ${ }^{5}$. Die Werke, aus denen die hier besprochenen Passagen entnommen sind, stammen aus verschiedenen historischen Epochen und Sprachstufen des Ägyptischen. Ich bin mir bewusst, dass die darin vorkommenden Phänomene nicht ohne weiteres zu vergleichen sind, da sie zwischen Altem Reich und Römerzeit Transformationen erfahren haben. Diese Zeilen können daher nur eine qualitative Analyse liefern.

Zur Erleichterung der chronologischen Einordnung sei hier eine grobe Übersicht zur Datierung der ägyptischen Sprachstufen gegeben, in denen die hier zitierten Literaturwerke verfasst sind:

- Mittelägyptisch (ca. 2000-1300 v. Chr.)

- Neuägyptisch (ca. 1300-700 v. Chr.)

- Demotisch (ca. 700 v. Chr.-500 n. Chr.) ${ }^{6}$

\section{Der Himmel kommt vom Himmel herab}

Dass Himmel und Erde zwei voneinander getrennte Sphären darstellen, ist communis opinio der alten Ägypter und wird in literarischen Texten mehrfach betont $^{7}$. Urheber dieser Trennung, dieses Hylems des „Hochhebens des Himmels“,

4 C. Zgoll 2019, 563.

5 Es sei kurz angemerkt, dass beim nicht-wissenschaftlichen Zugang der Präastronautik für Altägypten viel mehr Dinge als vom Himmel kommend betrachtet werden. Eine Auseinandersetzung mit solchen Thesen wird hier bewusst unterlassen.

Die Zitation von Quellen und Lemmata des ägyptischen Lexikons erfolgt nach dem Thesaurus Linguae Aegyptiae (TLA), http://aaew.bbaw.de/tla/, zuletzt besucht am 06.01.2017.

6 Texte können in hieroglyphischer, hieratischer und demotischer Schrift verfasst sein. Auf koptische und griechische Quellen wird hier nicht eingegangen. Es werden nur pagane Religionen betrachtet.

7 Dazu Jørgensen 2013, 91-94; Dorman 1999 und te Velde 1977, 161-170, besonders zu Konzepten von Weiblichkeit und Männlichkeit von Himmel und Erde, symbolisiert durch die Götter Nut und Geb; ikonographische Details zu Himmelsstützen bespricht Weyersberg 1961. 
ist der Schöpfergott ${ }^{8}$. Hinter dem Schöpfer ist zumeist der oberste Gott Re zu sehen. In den Klagen des Ipuwer wird sogar explizit auf die Trennung von Himmel und Erde durch die Götter abgehoben (Kol. 12, Z. 11-13):

nb-tm jri.n=f jwd p.t r zztw snd ḥ̂r ḥrnb $\{y\}<j y i>=f$ jr $m$ jrr=f st m ph.tj n n-m [']q=f r=s [m] msdd=k nḥm ḥw sj3

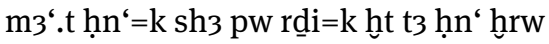
$\{$ hnn.w $\}<$ hnnn.w $>$
The Lord of Everything made the separation of the sky from the earth, (but) fear is on every face when he comes (?). When he does it as one who would attack us, who will treat it, [when] you disdain to save? Utterance, Perception, and Truth are with you, (but) what you would put throughout the land is uproar, together with noise and tumult!

Diese Passage ist Teil einer Klage, in der Ipuwer schlimme Zustände und eine Umkehr der gesellschaftlichen Verhältnisse prophezeit. Daher verwundert es nicht, dass dem Kommen des Allherrn, des Schöpfergotts, mit Schrecken entgegengeblickt wird. Die Trennung von Himmel und Erde wird einerseits positiv als Akt der Schöpfung, aber auch negativ als Trennung zwischen Götterwelt und Menschenwelt interpretiert, weil der Schöpfer sich enttäuscht von den Menschen zurückzog 9 .

Um diesen Schöpfungsakt zu feiern, gab es im ägyptischen Kalender das Fest des „Hochhebens des Himmels“. Es existierte aber auch eine allgemeine Kulthandlung dazu, die nicht an ein Festgeschehen geknüpft war, um den Fortbestand der Trennung des Himmels und der Erde sicherzustellen ${ }^{10}$.

Die intakte Trennung zwischen Himmel und Erde ist das Ideal der Weltordnung nach altägyptischer Vorstellung, der Maat. Die Menschen hatten ihren Platz in diesem Gefüge zu kennen, wie eine kurze Sentenz in der Lehre des Anchscheschonqi, Kol. 25, Z. 12 f aussagt: „Kenne das Fundament des Himmels! Kenne das Fundament der Erde!“ Dies steht innerhalb des Weisheitstexts, um dem Menschen seine Grenzen aufzeigen: Er soll die Hierarchien und den ihm zugeordneten Platz in Beruf und Gesellschaft erkennen, so wie irdische und himmlische

8 Belege dafür finden sich in folgenden Weisheitstexten: Lehre für Merikare, P. Carlsberg 6, § 131, Kol. X+5, Z. 4; Parallelen P. Moskau inv. 4658, Kol. 8, Z. 9-16 und P. Petersburg inv. 1116 A verso, Kol. 11, Z. 10-12.8 und in den beiden demotischen Lehren P. Brooklyn inv. 47.218.135, Kol. 3, Z. 18 und P. Insinger, Kol. 31, Z. 24-32.17. Im Zweiten Setna-Roman, P. BM EA inv. 10822, Kol. 5, Z. $8 \mathrm{f}$ wird dem Gott Thoth diese Rolle zugeschrieben.

9 Vgl. den Kommentar bei Enmarch 2008, 188 f.

10 Belege und Diskussion bei Kurth 1975, 136-146. 
Sphäre voneinander getrennt sind. Ein göttlicher Urheber, der die Fundamente geschaffen hat, wird nicht genannt. Wir können aber davon ausgehen, dass ein hoher Schöpfergott wie der Sonnengott Re dahinter verstanden werden soll.

Die Extremversion dessen, was vom Himmel kommen kann, ist der Himmel selbst. In zwei literarischen Quellen wird dieses Unglück erwähnt, wenn die Weltordnung komplett zusammenbricht: durch Krieg. Das erste Textbeispiel ist jedoch umstritten, da es in den zwei vorliegenden Versionen etwas unterschiedlich dargestellt wird. Der Himmel, so heißt es nach einer Maxime in der mittelägyptischen Lehre für Merikare, könne durch Aufständische zerstört werden, aber das dauere 100 Jahre (P. Carlsberg 6, § 113, Kol. x+3, Z. 9; Parallele P. Petersburg inv. 1116 A verso, §113, Kol. 10, Z. 8):

jri gr.w? bšt=k ḥdi.t p.t pw rnp.t 100

mn.w pw $\mathrm{m} \underline{\mathrm{d}} 3<\mathrm{y} . \mathrm{wt}>$ oder $\{\mathrm{m}\}<\mathrm{w}>\underline{\mathrm{d}} 3$
Handle doch, (damit) der gegen dich Aufständische schweigt! (?) Es braucht 100 Jahre, den Himmel zu zerstören (oder: zerfallen zu lassen).

Es ist ein Denkmal in (einer Zeit von) Unrecht. (?)

Es wird u. a. postuliert, dass das Zerstören des Himmels als Metapher für die Zerstörung des Andenkens an einen Menschen gemeint ist - dies ist aber nur eine Deutungsvariante ${ }^{11}$. Wenn man von dieser Interpretation ausgeht, lässt sich konstatieren, dass es sich mit dem Himmelssturz um ein negatives Ereignis handelt, für das in der Lehre Strategien zur Vermeidung gegeben werden. Mit den Aufständischen können Feinde des Pharaos Merikare, aber auch ganz allgemein Rebellen gegen den Sonnengott Re als Subjekte dieses Hylems gemeint sein.

Etwas konziser erwähnt ein demotischer narrativer Text die Möglichkeit, dass der Himmel auf die Erde kommen kann. Im Kampf um den Panzer des Inaros, P. Krall, Passage Kol. 9, Z. 18 spricht ein Fürst namens Pekrur vor dem Pharao, dass er dessen Feind Ur-di-imen-niut Krieg androhen will ${ }^{12}$ :

[iw] [=k] [(r)] [nwe] r t3 p.t r-iw=s pht hrr p3 t3 irm p3j=f g3j (n) mnmn
[Du wirst] den Himmel [sehen], indem er auf die Erde niedergeworfen ist, und ihr (der Erde) Beben.

11 Vgl. die Anmerkungen von Peter Dils im TLA zu dieser Passage, der die verschiedenen Emendierungen, Deutungsmöglichkeiten und die Forschungsdiskussion zusammenstellt.

12 Hoffmann 1996, 220, Anm. 1133 mit einer Parallele aus dem MYTHOS ÜBER HORUS UND SETH. 
Es ist interessant, dass die Drohung, den Himmel auf die Erde herabzuwerfen, von Vertretern der Partei des Pharaos kommt und nicht von deren Feinden. Der demotische Terminus ist pht, was wörtlich „niederwerfen; beugen, sich beugen“ bedeutet und beispielsweise in divinatorischen Texten vorkommt, wenn sich der mantische Spezialist oder das Medium über eine Schale beugt o. ä. ${ }^{13}$ Die Erzählkette aus Schildkampf, Beben der Berge, Niederwerfen des Himmels, Erdbeben, Kampf der Heere wie Stiere und Löwen und heißes Eisen zeigen eine Abfolge der gewaltvollen Auseinandersetzung auf, die vielleicht das Stilmittel der Steigerung darstellt. In jedem Fall handelt es sich um eine relativ ausführlich vorgebrachte Kriegsrhetorik, um die Entschlossenheit zum Kampf zum Ausdruck zu bringen. Vermutlich sollten dadurch auch andere in der Gegenwart des Königs und der König selbst überzeugt und angefeuert werden. Die beteiligten Personen und der Ort sind durch den Kontext der Erzählung klar, so dass man davon ausgehen kann, dass es sich um ein einmaliges Ereignis handelt. Der Konflikt in dieser Erzählung wurde durch Dämonen entfacht, die im Auftrag des Gottes Osiris den Krieg anzetteln.

Das Zusammenbrechen der Welt und damit des Friedens steht als Bild für die entfesselte Gewalt des Krieges, die die Aufständischen gegen den Pharao bringen (Lehre für Merikare) bzw. die Verbündeten des Pharao gegen dessen Feinde anwenden (Kampf um den Panzer des Inaros). Der Charakter des Hylems des herabstürzenden Himmels ist negativ für diejenigen, die davon betroffen sind. Im Text wird jedoch nicht thematisiert, dass die, die damit drohen, auch Schaden nehmen könnten. So ist davon auszugehen, dass es sich um ein lokales Ereignis handelt, das nur einen bestimmten Personenkreis trifft. Das Hylem des herabfallenden Himmels ist vermutlich in seinen Auswirkungen mit denen von Krankheitsbringern zu vergleichen: es ist kein globales Phänomen, sondern trifft einzelne ${ }^{14}$.

\section{Himmelskörper und Meteoriteisen kommen vom Himmel herab}

Etwas Handfestes, das von Himmel kam, ist Eisen. Seit dem vierten vorchristlichen Jahrtausend finden sich Belege für die Eisenverarbeitung, aber nur sehr

13 Vgl. das Lemma im TLA; Erichsen 1954, 139.

14 Vgl. auch die Beiträge von Jäger, Himmelsschilde und Blitze und Zomer, Demons and Tutelary Deities from Heaven in diesem Band. 
spärlich. Bereits ca. 3200 v. Chr. wurde das Metall zu Schmuckstücken verarbeitet. Das älteste bekannte Stück ist eine Perle einer Kette aus dem Grab eines hochrangigen Mannes in Gerzeh. Forschungen haben ergeben, dass dieses Eisen (Hämatit) meteoritischen Ursprungs ist ${ }^{15}$. Erst ab dem 6. Jahrhundert v. Chr. wurde Eisenerz in größeren Mengen abgebaut und verhüttet - im Unterschied zu den Hethitern, die dies bereits im 15. Jahrhundert v. Chr. vollbrachten ${ }^{16}$. Zuvor bediente man sich Eisen meteoritischen Ursprungs. Ab dem Neuen Reich wurde Eisen, ägyptisch bi3, auch als bi3-n-p.t bezeichnet, wörtlich „Eisen vom Himmel“ oder „Meteoriteisen“ genannt. Das Wort wird im Hieroglyphischen am Ende auch mit dem Klassifikator „Himmel“ geschrieben: $1 / 1=011^{17}$. Auch in dieser Zeit wird das Eisen aus dem Meteoriteneinschlag noch verwendet, z. B. bei einer Messerklinge aus dem Grab des Tut-anch-amun ${ }^{18}$.

Es ist auffällig, dass und fraglich, weshalb diese Bezeichnung auf einmal verwendet wurde. Die älteren literarischen Texte erwähnen diesen Begriff nicht, sondern verwenden nur bi3. Die demotischen Belege hingegen führen allesamt das Lemma bnpj, was auf bi3-n-p.t zurückgeht ${ }^{19}$. Warum hat sich die Bezeichnung erweitert? Es kann nicht auf einen erneuten Meteoriteneinschlag zurückgehen, da dieser bereits ca. 3400 v. Chr. geschah. Oder erinnerte man sich dessen um 1400 v. Chr. und betrachtete das Eisen vielleicht als göttliche Gabe, weil es vom Himmel kam? Liegt es vielleicht am Glanz des Eisens, der an die Farbe des Himmels erinnert ${ }^{20}$ ? In späteren Zeiten bestand die Vorstellung, dass die Götter goldene oder silberne Knochen haben; der Gott Seth eiserne. Man hat versucht, dies mit so genannten "fossil bones" aus dem Neuen Reich von der Nekropole von Qau el-Kebir in Mittelägypten zusammenzubringen ${ }^{21}$. Zudem ist verwunderlich,

15 Johnson/Tyldesley/Lowe/Withers/Grady 2013.

16 Yalçın 2005.

17 Zuvor wurde das Wort nur bi3 geschrieben. Vgl. Johnson/Tyldesley/Lowe/Withers/Grady 2013, 1003.

18 Comelli et al. 2016.

19 Helck 1995.

20 So in mesopotamischen Quellen, vgl. Johnson/Tyldesley/Lowe/Withers/Grady 2013, 1003.

21 Johnson/Tyldesley/Lowe/Withers/Grady 2013, 1004: "In later times, certain materials were linked to the gods, such as gold representing the flesh of the gods and the 'iron bones of Seth' as documented by the ancient historians Plutarch and Diodorus.” Auch in den Pyramidentexten des Alten Reichs gibt es die Vorstellung eiserner Knochen des Pharaos. Vielleicht bieten die wie Eisen glänzenden, mineralisierten Fossilien einen Anhaltspunkt, warum man das dachte. Auch die Verbindung von Feuerstein und Eisen mit Seth ist bedenkenswert, wie Graves-Brown 2007, 112-114 ausführt. 
weshalb die Ägypter erst relativ spät mit der massenhaften Bearbeitung und Verhüttung von Eisen begannen ${ }^{22}$. Eisenerzvorkommen gab es reichlich, v. a. im Sinai. Sie nutzten vor allem Kupfer und Bronze. Selbst Gold wurde häufiger verwendet. Man kann darauf schließen, dass Eisen als hochwertig galt, vielleicht mit einer bestimmten Bedeutung aufgeladen war. Eisenklingen wurden z. B. im Mundöffnungsritual zur Belebung der Toten verwendet. Nur reichliche 20 sicher datierbare Artefakte sind bisher bekannt ${ }^{23}$.

Es existiert aber leider kein (mythisches) Hylem, was sich auf die himmlische Herkunft des Eisens bezieht. Aber man könnte erwägen, ob der Begriff schon eine Handlung implizieren könnte: Eisen vom Himmel könnte bedeuten, dass jemand das Eisen vom Himmel geschickt hat. Sollte das Teil einer Erzählung gewesen sein, könnte in diesem kleinen Ausdruck „Eisen aus dem Himmel“ ein Mythos gesteckt haben. Die Motivation, warum Eisen um 1400 v. Chr. plötzlich als „Eisen vom Himmel“ bezeichnet worden ist, bleibt unklar. Vielleicht bringt ein neues Forschungsprojekt mehr Licht ins Dunkel ${ }^{24}$.

Ein Meteorit oder Komet, der auf die Erde fällt, ist Thema in der mittelägyptischen Geschichte des Schiffbrüchigen: Darin berichtet ein Schlangengott dem Schiffbrüchigen auf einer einsamen Insel, wie einst ein herabfallender Komet die Kinder und Verwandten der Schlange tötete (Z. 125-132):

sdd $d=j$ r $=f n=k$ mj.tt-jr.j h hpr.w m jw pn $w n=j$ jm=f ḥn'sn.w=j hrrd.w m-q3b=sn km.n=n ḥf3.w 75 m ms.w=j ḥn' sn.w=j $n n$ sḩ3=j n=k z3.t kt.t jni.t $n=j$ m sš3? ' $h$ '.n sb3 h3i.w pri.n n3 $\mathrm{m}$ x.t m-' =f hpr.n r=s nn wj ḥn` 3m.nj nn wj mhrr(.j)-jb=sn ' $h^{\natural}: n=j$ m(w)t.kw n=sn gmi.n=j st m hasy.t w'.t
Ich will dir nun Ähnliches berichten, das auf dieser Insel geschah, auf der ich weilte zusammen mit meinen Geschwistern, darunter (auch) Kinder. Mit meinen Kindern und meinen Geschwistern waren wir insgesamt 75 Schlangen. Ich werde dir nicht (meine) kleine Tochter nennen, die mir in weiser Voraussicht (? oder: mittels Gebet?) gebracht (?) wurde. Da fiel ein Stern herab. Durch ihn gingen sie in Flammen auf (d.h. sie verbrannten). (Dies) geschah aber, als ich nicht dabei war.

22 Johnson/Tyldesley 2014, 10.

23 Johnson/Tyldesley 2014, 13; genaue Liste in Johnson/Tyldesley 2016, 408-423.

24 Britisches Forschungsprojekt "Iron from the Sky" an der Universität Manchester, vgl. https://www.sciculture.ac.uk/project/iron-from-the-sky-the-science-and-culture-of-iron-in-ancient-egypt/, besucht am 02.01.2017. 
Sie verbrannten, als ich nicht in ihrer Mitte war. Da war ich zu Tode (betrübt) ihretwegen, als ich sie in der Form eines einzigen Leichenhaufens vorfand.

Obwohl die Schlange eine Gottheit ist, konnte sie das Unglück nicht verhindern, das höhere Mächte über sie und ihre Familie brachten. Das Hinunterfallen des Komets impliziert die Vorstellung eines göttlichen Verursachers der Katastrophe, der aber nicht namentlich genannt wird. Es ist auch nicht klar, ob die Schlange sich etwas zu Schulden kommen ließ und damit göttliche Bestrafung auf sich zog. Das Ereignis des herabfallenden Sterns ist eindeutig negativ konnotiert. Im Rahmen der Narration handelt es sich um ein einmaliges, auf die Insel der Schlange beschränktes Hylem.

\section{Dämonen und Krankheiten vom Himmel}

Weitere Boten, die hier erwähnt werden müssen, sind Dämonen ${ }^{25}$. In allen Belegen in literarischen Texten, in denen Dämonen als Hylemsubjekte vorkommen, agieren sie nicht von sich aus, sondern stehen unter der Kontrolle übergeordneter Götter. Dies geschieht u. a. im Rahmen der „Seuche des Jahres“, einer nicht spezifisch zu bestimmenden Krankheit, worunter man sich pestähnliche Symptome vorstellen kann. Die Erreger wurden laut Mythologie von der Göttin Sachmet selbst oder durch Dämonen vom Himmel auf die Erde geschickt. Die literarischen Quellen erwähnen Sachmet vor allem als mächtige, kriegerische Gottheit, mit der sich Krieger verglichen, aber es gibt auch Belege, in denen auf die „Seuche des Jahres“ abgehoben wird ${ }^{26}$.

Weitere Fälle in den literarischen Texten, in denen Götter Dämonen auf die Erde senden, sind in der Rahmenhandlung des in Demotisch verfassten Ersten Setna-Romans, wo der oberste Gott Re dem Gott Thoth erlaubt, sich an Na-Nefer-

25 Vgl. den Beitrag von Zomer, Demons and Tutelary Deities from Heaven und den Auswertungsbeitrag von A. Zgoll, Wer oder was vom Himmel kommt in diesem Band.

26 Vgl. mit neuerer Literatur Theis 2014, 174 f; erwähnt in Sinuhe, P. Berlin P 10499 aus Theben-West, 67-69; Parallelen P. Berlin P 3022 und Fragmente P. Amherst m-q (B), Z. 43-45; O. Ashmolean Museum inv. 1945.40, Z. 25 f; Beredter Bauer, P. Berlin P 3023 + P. Amherst 1, Version B1, 142-152; P. Ramesseum A = P. Berlin P 10499, recto, Version R, 22, 7-23, 2; Verkommener Harfner, Kol. 2, Z. 17-20. Vielleicht gehört dazu auch der milchige Krankheitserreger in der Geschichte mit Bes, Kol. x+3, Z. 24ff., vgl. Hoffmann/Quack 2007, 55-59. 
Ka-Ptah zu rächen. Dieser hat das geheime Zauberbuch des Thoth entdeckt, das der Gott auf Erden versteckt hatte. Nun sieht er die Möglichkeit zum Gegenschlag und schickt dafür Dämonen als seine Vollstrecker auf die Erde (P. CG Cairo 30692, Kol. 4, Z. 7 f):

$\underline{\mathrm{d} d}=\mathrm{w} \mathrm{n}=\mathrm{f}$ iw=f i.ir-ḥr=k irm rmt nb ntj $\mathrm{mtw}=\mathrm{f} \underline{\mathrm{dr}}=\mathrm{w}$ wt=w w' ht $\mathrm{t}$ r-hor $\mathrm{n}$ t3 p.t dd m-ir dj.t šm n3.w-nfr-k3-pth r mn-nfr $\mathrm{iw}=\mathrm{f} \mathrm{w} \underline{\mathrm{d}} 3 \mathrm{irm} \mathrm{rmt} \mathrm{nb} \mathrm{ntj} \mathrm{mtw}=\mathrm{f} \underline{\mathrm{d}} \mathrm{r}=\mathrm{w}$
Es wurde ihm (dem Thoth) gesagt: „Er soll in deiner Hand sein (wörtl. er ist vor dir) zusammen mit allen Leuten, die bei ihm sind." Ein Cheti-Dämon wurde vom Himmel herabgesandt: „Laß nicht zu, daß Naneferkaptah zusammen mit allen Leuten, die bei ihm sind, heil nach Memphis kommt!“

Eine ähnliche Situation gibt es in der Erzählung Der Kampf um den Panzer des Inaros. Die Götter sind verärgert und schicken Dämonen auf die Erde, damit sich die Menschen zerstreiten und es Krieg gibt (P. Krall, 1, 4-1.8) ${ }^{27}$. Da es sich aber um Osiris handelt, steht zu vermuten, dass die Dämonen aus der Unterwelt kommen. Vergleicht man beide Episoden, so zeigt sich, dass beide relativ ausführlich verfasst sind, mit konkreten Orten, göttlichen Urhebern und menschlichen Protagonisten. Es handelt sich jeweils um einmalige Ereignisse. Das Rachemotiv ist klar benannt.

Weil dies eine realistische Gefahr war, warnen Weisheitstexte vor den schändlichen Werken der Dämonen und unterscheiden diese dabei auch nach ihrem Aufgabenfeld oder Geschlecht. So heißt es beispielsweise in der demotischen Lehre des Papyrus Insinger, Kol. 8, Z. 18 f:

t3 wp.t n mw.t H.t-Ḧr t3 ntj hpr iwt n3 sḥm.wt i.ir špšj.t wr3.t ḥr p3 t3 h̆n n3 sḥm.wt
Das Werk der Mut (und) der Hathor ist es, was unter den Frauen geschieht. In den Frauen sind der gute und der böse Dämon auf Erden wirksam.

Ich habe diese Textstelle ausgewählt, weil die göttlichen Urheberinnen (Mut und Hathor) genannt werden und mit der postulierten Wirksamkeit auf Erden impliziert wird, dass die Dämonen aus dem Himmel kommen. Beide Göttinnen stehen für weiblich besetzte Lebensbereiche: Liebe und Mütterlichkeit. Frauen gehörten nicht zur vordergründigen Zielgruppe dieser Texte. Die knappe Lehrsentenz

27 Hoffmann 1996, 132. 
deckt sich mit der allgemein negativen Perspektive auf Ehefrauen, Geliebte, fremde Frauen etc. in der ägyptischen Weisheitsliteratur ${ }^{28}$.

\section{Götter besuchen ihre Tempel und die Menschen}

\subsection{Götter kommen an Festtagen auf Erden}

Götter, denen ein Tempel ${ }^{29}$ geweiht ist, besuchen diesen regelmäßig zur Abholung von Opfergaben oder an ihren Festtagen - und kommen dafür vom Himmel herab (oder alternativ aus der Unterwelt hinauf ${ }^{30}$ ) auf die Erde. Sie wohnen dann in ihrer Statue im Allerheiligsten ein. Manche dieser Götterbilder sind portabel für Prozessionen. Zu diesen Zeiten können die Statuen z. B. für Orakel oder andere divinatorische Praktiken konsultiert werden ${ }^{31}$. In der neuägyptischen Lehre des Ani wird vor der Macht des Gottes, der auf die Erde gekommen ist, gewarnt (P. Boulaq 4 ro, Kol. 20, Z. 14-17; Parallele P. Saqqara EES 75-S 45 = SCA 6319, Z. 10-13):

j:nw $\langle$ m $>$ jr.t $<$.t $>=k$ r p $3 y=$ f shr.w qnd $\mathrm{mtw}=\mathrm{k}\{$ snntj-tw $\}<\mathrm{sn}>\langle\mathrm{t} 3>\mathrm{m} \mathrm{rn}=\mathrm{f}$ sw (ḥr) di.t b3.w $<\mathrm{m}>$ ḥh n j3rw $<\mathrm{r}>$

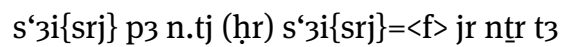
pn $\{\mathrm{n}\}$ p3 šw $<\mathrm{m}>$ hrr(.t) jw nzy=f tjtwy hrr-tp t3 d. d..tj sntrj m k3y=st m-mn(.t) $<\mathrm{r}>\mathrm{s} 3 \mathrm{rd} \mathrm{nb}\{\mathrm{t}\}-\mathrm{h}^{\mathrm{c}}$
Halte dir seine Art, zu zürnen, vor Augen (wörtl.: Achte <mit> deinem Auge genau auf seine Art, zu zürnen), (und) küsse die Erde in seinem Namen! Er zeigt (seine) Macht <in> Millionen von Formen, <um> den groß zu machen, der ihn groß macht. Was den Gott dieses Landes angeht, (das ist) das Licht/ der Lichtgott <im> Himmel, wobei seine Statuen auf Erden sind. Möge Weihrauch täglich als ihre Nahrung gegeben werden, <um> den Herrn der Erscheinungen/des (Sonnen-)Aufgangs zu stärken.

28 Dieleman 1998. Zur Stelle Lichtheim 1983, 162.

29 Weitere Güter, die vom Himmel kommen können, sind Baupläne der Tempel - vgl. dazu den Auswertungsbeitrag von A. Zgoll, Wer oder was vom Himmel kommt im vorliegenden Band.

30 Z. B. Hathor zu ihrem Senut-Fest in P. Vandier 4, 9-16, wo sie sich die Opferbrote der Menschen abholt und wieder mit in die Unterwelt nimmt.

31 Naether 2010, 38-44.359-410. 
Ani, der Schreiber des Tempels der Ahmes-Nefertari, spricht hiermit direkt seinen Schüler Chonsuhotep an, die Macht des Gottes nicht zu unterschätzen, der seine Macht über die Kultstatue ausübt ${ }^{32}$. Neben den Festtagen kamen die Götter aber auch zu anderen Zeiten in ihre Tempel. Das Tägliche Kultbildritual wurde dafür vollzogen, um für ihre Besuche bereit zu sein ${ }^{33}$. Vermutlich lag auch ein Festtag vor $^{34}$, als Amun in der Erzählung über den Aufstieg und Fall des Priestergeschlechts vom demotischen Papyrus Rylands 9 in seiner Statue einwohnte (Kol. 24, Z. $1 \mathrm{f})$ :

h.t $n$ nз ḥs.w i:wn-n3.w imn ir=w ndr.t wn-n3.w-iw=f ph r nzj wdj.w i:nqr $=w[i] w=f n^{\prime} j$ r p $3 w^{\prime} b=f n$ t $3 j=f$

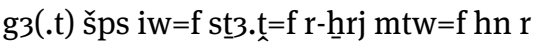
p3 mr-ḥs

\begin{abstract}
Abschrift der Lieder, die Amun machte, als er zu diesen ausgemeißelten Stelen gelangte, indem er sich in das Allerheiligste seines erhabenen Schreines begab, indem er sich hinabwandte und dem Vorsteher der Sänger zunickte.
\end{abstract}

Amun kommt vielleicht auf Erden, um mit Klageliedern gegen all das Unheil zu protestieren, das der Familie des Petese über drei Generationen widerfahren ist. Das Nicken zum Priester steht jedoch zweifelsfrei für ein Orakel der Prozessionsstatue $^{35}$. Auch diese Passage spricht von der Bewegung der Götter vom Himmel auf die Erde um zu richten und Rat zu erteilen. Die Quelle ist recht vage bei einer zeitlichen Angabe. Die Motivation für die Klagelieder liegt in dem göttlichen Zorn. Obwohl das Kommen des Gottes vom Himmel auf die Erde für ein Orakel eine allgemeine, wiederkehrende Situation darstellt, ist diese Handlung vor allem aus der Geschichte des Petese her zu erklären: der Gott war unzufrieden, wie mit seinem Priester Petese und dessen Nachfolgern und deren Familien umgegangen worden ist. Amun ist dabei nicht eine allgemeine Chiffre für den Schöpfer- und Hochgott, sondern der Amun von Teudjoi: der Stadtgott am wesentlichen Ort des Geschehens. Als Schöpfergott ist sein traditioneller Platz am Himmel. Er wird oft mit dem Sonnengott zu Amun-Re kombiniert.

32 Vgl. Quack 1994, $175 \mathrm{f}$.

33 Braun 2013, 244-253.

34 Vittmann 1998, 593-597.

35 Ritner (1993, 215) deutet es als eine magische Praxis (pḥ-ntr); Vittmann (1998, 593 f) sieht dies kritisch. M. E. muss man von einem Orakel der Prozessionsstatue ausgehen - das Nicken ist ein terminus technicus dafür und muss nicht mit einem pḥ-nțr in Zusammenhang stehen. 


\subsection{Götter kommen zu speziellen Anlässen auf die Erde oder überbringen etwas}

Eine bekannte mythische Erzählung ist die von der Himmelskuh ${ }^{36}$. In diesem auf Neuägyptisch überlieferten Text rebellieren die Menschen gegen den Sonnenund Schöpfergott Re. Dieser ist betrübt und will daraufhin den Kosmos neu ordnen. Teil dessen ist es, dass er seine Tochter Hathor, die Himmelskuh, auf die Erde schickt, um die Rebellen zu töten. Dann entwickelt Re Mitleid mit den Menschen und beschließt, das Massaker zu beenden. Er veranlasst, dass Bier durch Ocker rot gefärbt wird. Die blutrünstige Hathor trinkt die Flüssigkeit, wird betrunken und damit ist die Vernichtung des Menschengeschlechts abgewendet. Danach organisiert Re die Trennung von Himmel und Erde und verteilt Zuständigkeiten an einzelne Götter ${ }^{37}$.

Wenn die Götter vom Himmel kommen, sind sie nicht immer sofort als solche zu erkennen. In einer Erzählung Die Rache der Isis fliegt ein Skarabäus-Käfer zu den Menschen, der sich nicht als der Sonnengott, den er normalerweise darstellt, sondern als der vergöttlichte Baumeister Imhotep entpuppt (P. Saqqara dem. 1, 2, Kol. 6, Z. 18-20):

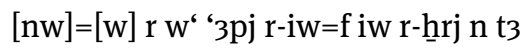
p.t $w 3 h=f$ i.ir-hir $=w$... nw.w nhs=f nw ir.t.t. $=w$ nw=w r $[\mathrm{Ij}-\mathrm{m}-\mathrm{h}$ tp $] \mathrm{st3}]$ [.t. $]=[\mathrm{f}]$ [s?] ... rk=f s ... mtw $=\mathrm{f}$ ir $\mathrm{p} 3 \mathrm{j}=\mathrm{f} \mathrm{smt}$ [ḥms]=f ḥr w` ift $r$ nz iwf.w
Sie erblickten einen geflügelten Skarabäus, der vom Himmel herabkam. Er (der Sonnenkäfer) landete vor ihnen. [...] Blicke. Er erwachte. Ihre Augen sahen. Sie sahen Imhotep. [Er] wandte [sich um(?) ...] Er wandte sich um (o. ä.) [...] und daß er dementsprechend tue (? o. ä.). Er saß auf einem Steinblock, während der Körper [.......].

Die Geschichte ist nicht nur aufgrund der vielen Lücken mysteriös. Man kann erahnen, dass die kranke Frau ein Problem mit der Göttin Isis hat, die einen Menschen aus Rache umgebracht hat. Sie fleht im Kreis der Priesterschaft den Halbgott Imhotep um Hilfe $a^{38}$. Dass der Halbgott auf die Erde kommt und dann erst

36 Vgl. Hornung 1982.

37 Guilhou 2010.

38 Smith/Tait 1983, text 2 front, 97 f, Anm. Sigma: der Skarabäus ist Imhotep, s. S. 105 zu Imhoteps Rolle in der Geschichte. 
nach einer Verwandlung seine Gestalt zeigt, ist ein einmaliges, numinoses Ereignis, das bei den antiken Zuhörern sicherlich nicht seine Wirkung verfehlt hat.

Ein ganz faszinierendes Artefakt ist das Zauberbuch des Gottes Thoth, das dieser auf Erden bringt und dort versteckt, als er mit anderen Göttern vom Himmel herabkommt. Dieses ist das Objekt, um das es im Ersten Setna-Roman geht. Als Setna, der älteste Sohn Ramses' II., ein gebildeter, aber auch verwöhnter Dandy, während einer Prozession des Gottes Ptah nichts Besseres zu tun hat, als Inschriften am Dromos zu lesen, sagt ihm ein plötzlich auftauchender, ominöser alter Priester (Kol. 3, Z. 12 f):

$[\mathrm{iw}=\mathrm{f}$ h $\mathrm{pr}][\mathrm{iw}]=[\mathrm{k}] \mathrm{wh} 3$ ‘ $\check{\mathrm{s}} \mathrm{sh}$ im $\mathrm{n}=\mathrm{j}$ $t w=j \underline{t} 3 j=w$ t=k r p3 m3' ntj-iw p3j dim“ n.im=f iw dhọtj p3 i.ir sh=f n dr.t.t.t=f h( $\left({ }^{\circ}\right)=f$ iw $=f$ n'.k r-hrij m-s3 n3 ntr.w
[Wenn] du (wirklich nützliche) Schrift(en) lesen willst, komm zu mir, daß ich dich zu dem Ort bringen lasse, an dem dieses Buch ist, das Thoth eigenhändig geschrieben hat, als er herunterkam im Gefolge der Götter!

Setnas Neugier ist geweckt und er findet das Zauberbuch, aber es bringt ihm und seiner Familie großes Unheil, denn der Gott Thoth ist nicht erfreut, dass ein Mensch seine Zaubersprüche anwendet. Die Schriftrolle mit den magischen Sprüchen ist ein handfestes Artefakt, das hier in Zusammenhang mit einer konkreten Handlung eines Gottes steht. Thoth ist nicht der höchste Gott, wird aber mit Magie, Schrift und Rechenkunst verknüpft und kommt mit seinem zentralen Kultort Hermoupolis Magna in den Geschichten um Setna oft vor. Das Buch ist jedoch in der Nähe des Ortes Koptos im Nil versteckt, wo es in sieben Truhen verstaut und von Schlangen und einem Uroboros als göttlichem Wächter beschützt wird. All dies hat Thoth veranlasst, aber es hält Na-Nefer-Ka-Ptah und später Setna nicht davon ab, die Schriftrolle, das Hylemobjekt, an sich zu nehmen. Der moderne Leser wundert sich, warum der Gott überhaupt vom Himmel kam und das Buch auf Erden versteckte. Folgende Dinge kann der magische Praktiker mit dem Buch in Erfahrung bringen (P. Kairo CG 30646, Kol. 3, Z. 12-15), darunter das Wissen über himmlische Angelegenheiten und die Götter als Sterne am Nachthimmel:

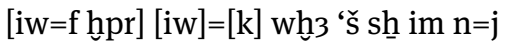
$t w=j \underline{t} 3 j=w$ t=k r p3 m3' ntj-iw p3j dm n.im=fiw Dḥwtj p3 i.ir sh=f $\mathrm{n}$ dr.t.t. $=f$ $h\left({ }^{\prime}\right)=f$ iw=f na.k r-hrij m-s3 nz ntr.w hp 2 n sh n 3 ntj hrr-3(.t).t=f iw=k [‘š] [p3] [hp] [mh-1] [iw] [=k] [r] [ph]re t3 p.t p3
[Wenn] du (wirklich nützliche) Schrift(en) lesen willst, komm zu mir, daß ich dich zu dem Ort bringen lasse, an dem dieses Buch ist, das Thoth eigenhändig geschrieben hat, als er herunterkam im Gefolge der Götter! Zwei schriftliche 
t3 t3 dw3.t n3 tww.w n3 jm.w iw=k r gm n3 ntj-[iw]? n3 ipd.w n t3 p.t irm n3 ddfe.w r dd.t $=w$ d dr=w iw=k r nw r n3 rjm.w n p3 mtr iw wn [mḥ-ntr] [21] [n] [mw] (n) [t3j] [=w] [rj.t]-ḥrj.t iw=k ‘š p3 hp mh-2 iw=f (r) ḩpr iw=k hn imnt iw=k n p3j=k gj hrr p3 t3 ' $n$ iw=k r nw r p3-r' iw=f h'.w n t3 p.t irm t3j=f psd.t irm p3 i'h [n] p3j=f gj n wbn [dd] [n] [=f] [n3.w-nfr-k3-pth]
Formeln sind darauf. Wenn du [die erste Formel rezitierst, wirst du] den Himmel, die Erde, die Unterwelt, die Berge (und) die Meere bezaubern. Du wirst alles verstehen, was die Vögel des Himmels und das Gewürm (der Erde) sprechen werden. Du wirst die Fische der Tiefe sehen, obwohl [21 Gottesellen Wasser] über [ihnen] sind. Wenn du die zweite Formel rezitierst, wirst du in der Unterwelt sein, indem du wieder in deiner Art auf der Erde bist. Du wirst Re schauen, wie er mit seiner Neunheit im Himmel erscheint, und den Mond bei seinem Aufgehen.

Dass dies dem Gott Thoth nicht recht ist, kann man sich leicht vorstellen. Die Menschen sollen keinen Einblick in dieses göttliche Geheimwissen haben. Er schickt daher Dämonen als seine Rächer auf die Erde.

Narrative Texte führen manchmal Götter als Protagonisten. Dies ist der Fall in der neuägyptischen Geschichte Astarte und das Meer, wo neben dem Pharao und seinen Bediensteten der Meeresgott Jam und die Göttin Astarte die Hauptrollen spielen. In einer Passage ist aufgrund der Lücken unklar, ob etwas vom Himmel kommt oder zu ihm aufsteigt. Es geht darin um Abgaben und Kriegsbeute, die das Meer als Tribut bekommt. In anderen Traditionen ist Astarte auch Partnerin des $\mathrm{Jam}^{39}$. Astarte als Überbringerin hat in der Erzählung einen recht passiven Charakter und wird zum Spielball der männlichen Protagonisten. Sie kommt aber aus dem Himmel, wie die Stelle Kol. 2, Z. x+18 f. belegt, wo sie von einem Gott oder von Jam gefragt wird:

['ḥ'].n [d] d. n=f n=s jy=[t] [tnw] t3 šrj.t

(n) Ptḥ t3 ntr(.t) [q]n[d].t (n) nšn.y.w
Da sagte er (= Gott oder das Meer) zu ihr: „[Woher] kommst [du], Tochter des

39 P. BN inv. 202 + P. Amherst inv. 9, Kol. 1, Z. x+5-x+10: ,[Und dann,] nach 40 (?) Tagen, da [---] der Himmel [---] hinabsteigen zum [---] das Meer. Dann [---, die Er]de schuf [---] die 4 Enden (wörtl.: 4 Ufergebiete) der [Erde ---] in seiner Mitte, als würde [---] hochheben (?) [---] [--- sein]en Herrscherthron - LHG. Dann [---] er [,er“ = Amenhotep II.] [---] trug Gaben zu ihm. [---] im/vor/als Gericht. Da trug (die Erntegöttin) Renenut [---] als Herrscher - Leben-Heil-Gesundheit - [---] Himmel“. Vgl. auch Ayali-Darshan 2010. 
jn-jw ng[3 $\left.\left\{{ }^{6} \mathrm{w} . \mathrm{t}\right\}\right]$ ? $=\mathrm{t}[\mathrm{n}] 3 \mathrm{y}=<\mathrm{t}>\mathrm{tjw} . \mathrm{wt}$ n.tj $\langle$ hr. .j? $>$ rd.wj $=\langle t>$ jn-jw $s d=\langle t>$ n3y=t ḥbs.w n.tj har=t m p3 šmi.t jy.t jri.n $=<t>m$ t3 p.t hin' $p_{3} t_{3}$
Ptah, du zürnende Göttin der Wütenden? Hast du <deine> Sandalen, die $<$ unter deinen $>$ Füßen sind, zerbrochen? Hast $<$ du $>$ deine Kleider, die an dir waren, bei deinem Herumwandern am Himmel und auf der Erde (wörtl.: bei dem Gehen und Kommen, das $<\mathrm{du}>$ am Himmel und auf der Erde machtest) zerrissen?“

Leider kennen wir die Antwort nicht, denn danach sind 22 Zeilen verloren ${ }^{40}$. Astartes Position am Himmel ist in Ägypten belegt; so wurde sie als „Mondauge“ bezeichnet ${ }^{41}$.

Ähnlichkeiten mit der Geschichte des Sterntalers ${ }^{42}$ hat eine kurze demotische Erzählung namens Der Beistand der Isis auf dem Krug B der Krugtexte. Dem Protagonisten, einem gewissen Chalamenti, wird übel mitgespielt - er hat keinen Besitz mehr, wird verprügelt und flieht aus der Stadt, bis es schließlich dunkel wird und er sich in seiner Not an die Göttin Isis wendet, die als Gestirn am Himmel erscheint (Z.19 f) ${ }^{43}$ :

hprr] grḥ r-ḥr=j ir=j ḍh ḥr nzi(=j) iwf.w $\mathrm{fj}<=\mathrm{j}>\mathrm{h} \mathrm{r}=\mathrm{j}\left[\mathrm{iw}=\mathrm{j}\right.$ nw r] $\mathrm{n} 3 \mathrm{~h} \mathrm{~h}^{\prime} \cdot \mathrm{w}$ n $3 \mathrm{~s} . \mathrm{t}$ wr.t mw.t ntr $t 3$ ntr.t. '3.t iw $=$ w hpr $r$

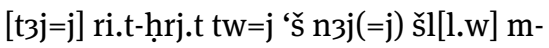
b3h [3s.t] wr.t [t3] ḥnw.t 3s.t wr.t [mw.t] ntr t3 ntr.t '3.t rh p3 hp t3 wpj.t [irm(?)] Hrr-wdי (s3) Hr-Lmn[nt p3 ḥtl] p3j=j sn hm
[Nun kam] die Nacht über mich und ich hatte Schmerzen(?) am (ganzen) Leib. Da wandte ich mein Antlitz [nach oben und erblickte die] Erscheinungen der Isis, der Großen, der Gottesmutter, der großen Göttin, wie sie oberhalb von mir waren. Ich rufe meine Gebete zur großen Isis: „Herrin Isis, Große, Gottes[mutter], große Göttin! Kenn mein Recht und mein Gericht [mit] Haryothes, (Sohn des) Chalamenti, meinem jüngeren Bruder!“

40 Nicht kommentiert in der Edition von Collombert/Coulon 2000.

41 Bonnet 1952, 55-57.

42 Grimm/Grimm 2002 ${ }^{19}$, 666-668.

43 Vgl. Thissen 2004. 
Die Klage ist von Erfolg gekrönt (Z. 20-22):

p3 h(r)w t3 wnw.t (n) dd p3 g3j i-ir mt hpr dj=j iw $\underline{d}[r . t]=j[r$ inh $] j($ ?) $i-i r=j$ gm ht-nbw inh $=\mathbf{j} d j=j$ iw dr.t=j m-s3=f dd $i w=j$ rh $f j=f d i=j h r r=j(r)$ ḥrj.t [3r] ${ }^{\circ} j$ tbh $=[j]$ tw=j ‘š(3j) n3j(=j) šll[.w m-b3h 3s.t wr.t t3] ntr.t '3.t d dd r-jr=t ḥh (n) ḥbsd t3j(=j) ḥnw.t 3s.t wr.t mw.t ntr [t3 ntr.t '3.t .... dj=t n=j hlt-nbw dj=w n=j p3 g3j fj n=j n-im=f m-s3 p3 g3j i-ir-bnr [h.s.t] $n-i m=f$
Als (ich) dies sagte, geschah es, daß ich meine Hand $\mathrm{zu}$ meinen Augenbrauen ausstreckte. Da fand ich ein goldenes Diadem (?) (an) meinen Augenbrauen. Ich streckte meine Hand aus, damit ich es nehmen könnte. Ich wandte mein Antlitz zur Herrin des [Ur]äus. [Ich] betete und machte mein Flehen zahlreich [vor Isis, der Großen, der] Gottes[mutter], der großen Göttin: „O mögest du Millionen Feste feiern! Meine Herrin, Isis, Große, Gottesmutter, mögest du Millionen Feste feiern! Meine Herrin, Isis, Große, Gottesmutter, [große] Gött[in, denn] du hast mir ein goldenes Diadem (?) gegeben. Möge (es) mir gegeben sein, es hochzuheben, nachdem es verlorengegangen war!“

Auch wenn die Geschichte hier zu Ende ist und wir nicht erfahren, warum genau Chalamenti Ärger mit seinem Bruder hat und weshalb das Diadem als verloren galt, so ist dieser Krugtext doch ein sehr treffendes Beispiel dafür, worum es uns in diesem Buch geht. Die große Göttin Isis lässt mit dem Diadem ein konkretes Artefakt als Hylemobjekt vom Himmel auf die Erde herunter. Im Text wird dies so präsentiert, dass das Diadem, eigentlich ein Herrschaftszeichen, von oben verliehen und auf dem Kopf des Chalamenti erscheint. Das Ereignis ist ein einmalig und positiv besetzter Akt: Isis hilft denen, die unverschuldet in Bedrängnis geraten sind. Das ist vermutlich auch die Moral, die sich für den Rezipienten der Geschichte erschließen sollte. Ich denke nicht, dass die Krone ein irdisches Artefakt ist, das Chalamenti vorher verloren habe o. ä. 


\section{Vom fruchtbaren Regen zur Fülle, die vom Himmel auf die Erde kommt}

Niederschläge wie Regen, Graupel, Hagel oder Schnee sind selten im östlichen Mittelmeer: allenfalls in den Küstenregionen zur Regenzeit im Winter, vor allem im Februar, treten sie auf ${ }^{44}$. Regen fällt in Tropfen aus Wolken auf die Erde, kommt also aus dem Himmel. Es verwundert daher nicht, dass ihm göttlicher Ursprung attribuiert wird bzw. dass Götter das Wetter kontrollieren können.

Im landwirtschaftlichen Kalender sind Regenfälle zu bestimmten Zeiten der Garant für ausreichende Ernten und damit die adäquate Versorgung der Menschen. Bleibt der Regen aus oder kommt er sintflutartig, kann dies zu katastrophalen Zuständen führen. Gerade solche anomalen Zustände waren in literarischen und dokumentarischen Texten erwähnenswert - zum Beispiel, wenn Regen im Übermaß fiel oder es im Sommer schneite ${ }^{45}$. All dies gefährdete menschliche Unternehmungen oder die Versorgung: die erwartete Fülle war in Gefahr, also musste eine höhere Macht angefleht werden, um das Gleichgewicht wiederherzustellen ${ }^{46}$. Dies konnten Götter, aber auch Herrscher sein. Manchmal wurden Mittler damit beauftragt, den Kontakt mit den höheren Mächten herzustellen und um Regen zu bitten. Darüber hinaus kann der Niederschlag auch übertragene Bedeutungen aufweisen (regnen als eine Art und Weise, wie etwas vom Himmel auf die Erde kommen kann) oder eine Begleiterscheinung göttlicher Handlungen $\operatorname{sein}^{47}$. Blitz und Donner können göttliche Aktionen verstärken.

Wenn man an herausragende Regen-Episoden der Antike denkt, fällt einem vielleicht das so genannte „Regenwunder“ auf einem Feldzug des römischen Kaisers Marcus Aurelius ein. Als die Römer 171-175 n. Chr. gegen die Quaden im Markomannenkrieg kämpfen, kommt es zu Wassermangel und damit großem Durst unter den Legionären. Der Kommandant Donatus betet daraufhin zum Gott der Christen um Rettung aus der misslichen Lage. Dieser lässt es daraufhin regnen.

Die Schilderung des Regenwunders findet sich hauptsächlich bei dem zeitgenössischen Historiographen Cassius Dio, Buch 71, Abschnitte 8-10 ${ }^{48}$. Dazu gibt

44 http://www.wetter-in-aegypten.de/, besucht am 28.12.2016.

45 Z. B. Dorn/Müller 2006, 90-93. Eine pittoreske Schilderung ist Jaggi 2008.

46 Vgl. für Ägypten Dorn 2016, 30-38; Bickel 2005; Quack 2012.

47 Vgl. den Beitrag von N. Jäger, Himmelsschilde und Blitze in diesem Band: Das ancile (Schild) in Ovids Fasti kommt nach dreimaligem Blitzen und Donnern Jupiters vom Himmel.

48 Konsultierte Edition: Gros 1870. Weitere Quellenkritik unternimmt Kovács 2008, 387-404. 
es auch eine bildliche Darstellung auf der Marc-Aurel-Säule in Rom. Dort lässt es ein großer alter Mann mit Bart und mit wie Flügel ausgebreiteten Armen vom Himmel auf die Soldaten herabregnen. Diese Episode wurde von Forschern als magischer Akt ägyptischen Ursprungs gewertet. Der Grund dafür ist in Cassius Dios Erzählung und in einer weiteren schriftlichen Überlieferung zu suchen: In dem spätantiken Lexikon Suda findet sich der Eintrag, dass Julianus Theurgistes oder der ägyptische Philosoph Arnouphis Urheber des Regenwunders seien ${ }^{49}$. Dies muss nicht im Gegensatz zum christlichen Gott als Regenbringer stehen könnte der Magier doch Gott mittels mantisch-magischer Techniken um Hilfe gebeten haben. István Tóth versucht, die ägyptische Prägung dieser Geschichte durch die Kulte ägyptischer Götter in der Donauregion stärker zu betonen (Thoth/Hermes als rettender Gott) ${ }^{50}$. Peter Kovács weist zu Recht auf eine Aussage Marc Aurels in dessen Meditationes hin, dass wir hinter dem Regengott Jupiter Pluvialis sehen sollen, der um Regen und Blitze gebeten werden konnte ${ }^{51}$. Es ist schwierig, die ursprüngliche Variante des Regenwunders herzustellen, da die Episode bereits in der Antike in unterschiedlichen Versionen, christlich interpoliert und in Zusammenhang mit einer weiteren Geschichte, einem Blitzwunder, vorlag $^{52}$. Was jedoch auffällt, sind zwei Punkte in dieser Hylemsequenz: die göttliche Intervention, d. h. die göttliche Urheberschaft des Regens, und die Anwesenheit des Kaisers, die in den Quellen gesondert erwähnt werden.

49 Suda, Lemma 'Iov入ıvós, Adler-Nummer: I 434, online verfügbar unter http://www.stoa.org /sol-bin/search.pl?db=REAL\&field=adlerhw_gr\&searchstr=I,434, besucht am 28.12.2016. Der griechische Text in englischer Übersetzung lautet: „On one occasion, when the Romans were suffering from thirst, [the story goes that] this man suddenly created and summoned up darkcoloured clouds and let loose heavy rain along with thunder and lightning bolts one after another. And this [they say that] by some cleverness Julian achieved. But others assert that it was Arnouphis, the Egyptian philosopher, who made this miracle.“

50 Tóth 1976, 102 zur Person des ägyptischen Magiers. Ältere Besprechungen sind Guey 1948; Posener 1951.

51 Kovács 2008, 395. Seiner Auffassung nach ist kein Mittler dafür nötig (S. 400.)

52 Kovács 2008, 389-397; Übersicht S. 403 f. 


\subsection{Lexikographische Annäherungen zum Regen}

Das ägyptische Grundlexem für „Regen“ ist ḥw.t, demotisch hww ${ }^{53}$. Es kann auch „Flut“ bedeuten. Weniger gebräuchlich ist snm „Regenflut“, ein Unwetter in Verbindung mit Wind ${ }^{54}$. Das Wort jgp „Regenwolke, Wolke“ ${ }^{55}$ konnte u. a. metaphorisch gebraucht werden ${ }^{56}$.

Des Weiteren gibt es eine Abwandlung, die auf den Himmel als den Ursprung des Regens hinweist: mw-n-p.t „Regen“, wörtlich „Wasser des Himmels“57. Dieser Begriff ist belegt in Papyrus Anastasi 4, einem Textträger der sogenannten Late Egyptian Miscellanies, einer Sammlung lehrhafter Sentenzen und Musterbriefe für die Schreiberausbildung etc. ${ }^{58}$ In einem Text namens Lob auf Piramesse, ein laus urbis auf die ägyptische Hauptstadt unter den Ramessidenkönigen, heißt es in Kol. 6, Z. 9:

bw ptrj=f mw-n.w-p.t jw $=$ f $<$ m $>$ b3.w (n) $\left(\left|W s r-m 3^{6} . t-r^{6}\right|\right) ' n h-(w) \underline{d} 3-s(n b)$ p3 k3 mri qn(.t)
Es (d. h. Chatti) sieht keinen Regen (d.h. es herrscht Dürre), weil es <in> der Macht(manifestation) <von $>$ (Kartusche|User-Maat-Re|Kartusche) Leben-Heil-Gesundheit ist, dem Stier, der Tapferkeit liebt.

Hier wird die Machtposition des Königs Ramses II. gepriesen, der die Fremdländer nur aufgrund seiner Gnade leben lässt. Es ist interessant, dass das Ausbleiben des Regens nicht Werk der Götter, sondern des Königs ist und dass die Vorstellung, dass der Regen vom Himmel kommt, durch die Verbindung mw-n-p.t ,Wasser des Himmels“ ausgedrückt wird.

Ähnlich konstruiert ist das Demotische Lexem hw-m-p.t „Regen des Himmels“, für das es aber nur einen Beleg in einem magischen Text gibt. Hier war es

53 Wb 3, 49.1-4; Erichsen (1954), 295. Wenn nicht anders erwähnt, wird dieses Wort in den zitierten literarischen Texten verwendet.

$54 \mathrm{~Wb}$ 4, $165.11 \mathrm{f}$. Der TLA führt nur zwei Belege auf.

$55 \mathrm{~Wb} 1,140.20 \mathrm{f}$.

56 Z. B. in der Formulierung „erobern wie ein Wolkenbruch“ als Umschreibung, dass der Pharao ein Gebiet blitzschnell einnimmt (Lehre für Merikare, P. Petersburg 1116 A verso, Kol. 7, Z. 3 f, § 72 f, die Parallele in P. Moskau 4658, Kol. 5, Z. 10 ist weniger gut erhalten).

57 Wb 2, 51.2. Vgl. dazu Leblanc 1995.

58 = P. BM EA inv. 10249 recto, Kol. 6, Z. 1-6.10. 
wichtig für die korrekte Anwendung eines Rezepts, frisches Regenwasser zu verwenden, damit der dazugehörige Zauber wirksam ist ${ }^{59}$.

Es fällt auf, dass Regen in Ägypten in Zusammenhang mit Flut gedacht wird. Niederschlag ist in den meisten Regionen Ägyptens selten und wird daher mit einem Naturphänomen verglichen, die man weitaus besser kennt: die alljährliche Nilüberschwemmung ${ }^{60}$. Serge Sauneron kann nachweisen, dass es sogar in griechischen und lateinischen literarischen Texten ein Topos ist, Ägypten gerade wegen der Absenz von Wolken und Regen als fruchtbares Land zu beschreiben wegen der Nilflut. Zum Teil wird der Nil als Gott gepriesen, zum Teil werden Regenfälle außerhalb Ägyptens für die Abundanz des Flusses verantwortlich gemacht ${ }^{61}$ - der Monsun. Es verwundert daher nicht, dass die Überschwemmung als Hapi vergöttlicht wurde - auch wenn der König oder andere Götter wie z. B. Seth, Baal oder Isis als Regenbringer gefeiert werden konnten ${ }^{62}$.

\subsection{Herrscher als Regenbringer}

Herrscher oder prominente Personen als Regenbringer sind in der griechisch-römischen Antike und darüber hinaus gut bekannt - Peter Kovács erwähnt Alexander den Großen, Sulla, Kaiser Hadrian und den Propheten Elias ${ }^{63}$. Auch der Pharao Taharqa ist mit einer solchen Episode verknüpft ${ }^{64}$. Auf die Fähigkeit Ramses' II., Anrainerländer zu strafen, indem man ihnen den Regen entzieht, wurde bereits weiter oben hingewiesen.

Schnee und Regen als unübliches Wetter wird auch in den Textzeugen von Ramses II. historischen Inschriften zur so genannten Ersten Hethitischen Heirat beschrieben, als sich der Pharao folgende Gedanken macht:

59 P. mag. London-Leiden Kol 20, Z. 22.

60 Jung 2007.

61 Sauneron 1952.

62 Schäfer 1931, 139 zu Regen und Donner am Geburtstag von Isis und Seth. Vielleicht findet sich in den Pyramidentexten ein weiteres Regenwunder (non vidi): Wilke 1931. Umstritten ist eine Regengöttin namens ḥw/ḥwj.t, wie sie in einer Version des Mythos von der Himmelskuh, in Z. 85 f im Grab Sethos’ I. (KV 17) vorkommt: „Der Ba der Ewigkeit ist (die Göttin) Regen.“ Weitere Belege gibt es nicht, vgl. LGG V 56b und die Anmerkung von Lutz Popko im Thesaurus Linguae Aegyptiae, ad locum.

63 Kovács 2008, 399.

64 Baud 2010. 


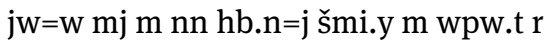
d3-h m n3.w hr.w n ḥwi.y s-r-q.w hpr.w $\mathrm{m}$ pr.t
Wie ergeht es (wohl) denjenigen (wörtl.: wie was sind sie, diese), die ich aussandte, denjenigen, die mit Aufträgen nach Djahi (= Gebiet in Palästina) gingen in diesen Tagen des Regens, wenn es (sogar) schneite in der peretJahreszeit (wörtl.: Schnee entstanden ist) ${ }^{65}$ ?

In der Folge opfert Ramses dem Gott Seth und ruft ihn mit folgenden Worten an:

p.t hir-'.wy=k\{j\} t3 harr rd.wy=k wd. pw hpr.wt nb mj tm=k jri.t hw.yt qb s$r-q . w$ r spr $n=j$ bys.wt $w d=k n=j$ nswtbj.tj wsr-m3'.t-r'-stp.n-R'w s3-R' R'msi-sw-mri.y-jmn di 'nh
Der Himmel ist unter deiner Aufsicht. Die Erde ist dir Untertan (wörtl.: unter deinen Füßen) Was du befohlen hast, verwirklicht sich vollends (wörtl.: ist all das, was geschieht), so wie du keinen Regen, eisigen Wind und Schnee schaffen sollst, bis die Wunder mich erreicht haben, die du mir zuweist, (nämlich) dem König von Oberägypten und Unterägypten Usermaatre-setepenre, dem Sohn des Re, Ramses-meriamun, dem Leben gegeben ist. (Z. $37 \mathrm{f}$ )

Mit den Wundern meint der Pharao, dass ihm sein Götterpatron Seth militärische Erfolge zubilligt, die nicht durch anomale Wetterereignisse gefährdet werden sollen. Daraufhin lässt Seth das Wetter wärmer werden, wie es zur Jahreszeit passt ${ }^{66}$. In einer mittelägyptischen Erzählung auf dem Papyrus Westcar befindet sich ein LEGITIMATIONSMYTHOS, der die Gottessohnschaft von bestimmten Königen des Alten Reichs belegen soll. Drei Königssöhne werden von einer menschlichen Frau im Haus ihres Ehemannes geboren, die durch den Gott Re gezeugt worden sind. Bei diesem Wunder assistieren Götter bei der Geburt. Danach schaffen sie drei Kronen für die späteren Herrscher und verstecken diese in einem Getreidesack. Dies wird begleitet von einem Regensturm (P. Westcar, Kol. 11, Z. 10-17):

65 So z. B. in den Inschriften von Abu Simbel, Großer Tempel, Fassade, Stele Jahr 34 (Erste Hethitische Heirat), Zeile $36 \mathrm{f}$.

66 Vgl. Borghouts 1984, 13-16 bzw. Kitchen 1996 (=KRI II), 249, 7/9-13/15. 
'h'.n dd.n 3s.t n nn ntr.w ptj n3 n.tt=n jyi.wjn $r=s n n$ jri.t bj(3).yt $n$ n3 $n(. j)$ hrd.w $s m j=n$ n p3y $=s n$ jt rdi jwi.t=n 'h'.n msi.n=sn h'.'w $3 \mathrm{n}$ nb 'nh-(w)d3-

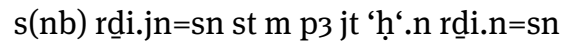
jwi.t p.t m d' hr hiw.yt ' $h^{\prime} \cdot n$ 'n.n=sn st r p3 pr 'h'.n dd.n=sn hạ 3 di=tn p3 jt ' $3 \mathrm{~m}$

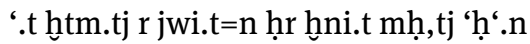
rdi.n=sn p3 [jt] m '.t htm.tj ' ha'.n rdi.n=sn p3 [jt] $m$ '.t htm.tj
Daraufhin sagte Isis zu diesen Göttern: „Was sind das für Gründe, derentwegen wir (weg)gegangen sind, ohne ein Wunder zu vollbringen für diese Kinder, so dass wir ihrem Vater berichten könnten, der veranlasst hat, dass wir kommen." Daraufhin schufen sie drei Kronen des Herrn (=Königskronen), Leben-Heil-Gesundheit, und legten sie dann in den (Sack) Gerste. Daraufhin ließen sie den Himmel (herab)kommen mit Sturmwind und Regen. Daraufhin wandten sie sich (wieder) um zum Haus. Daraufhin sagten sie: „Ach, möget ihr doch den (Sack) Gerste hier in eine Kammer, die verschlossen ist, legen, bis dass wir zurückgekommen sein werden vom Tanzen im Norden.“ Daraufhin legten sie den (Sack) Gerste in eine Kammer, die verschlossen wurde.

Die Götter Isis, Nephthys, Mesechenet, Heqet und Chnum sind hier die Verursacher des Regensturms. Die Funktion des Unwetters ist mir nicht ganz klar: Wollen die Götter vom Verstecken der Kronen ablenken? Oder wollen sie die Designation der späteren Herrscher durch ein Naturomen verstärken, damit die Menschen den Tag in besonderer Erinnerung behalten? Geht es ihnen darum, mit dem tosenden Wind und Regen ihren Worten Nachdruck zu verleihen? Man könnte auch daran denken, dass die Götter das Unwetter schicken, damit sie einen Grund haben, den Sack im Hausinneren zu deponieren.

\subsection{Götter als Regenbringer}

Die nächsten Textstellen verdeutlichen ebenso die Kraft, die mit Regen, Sturm und Gewitter verbunden wird. In der mittelägyptischen Lehre des Papyrus Ramesseum 2, Kol. 1, Z. 6 erfahren wir vermutlich von der Wut des obersten Gottes, der er mit heftigen Niederschlägen Ausdruck verleiht: 
... ]nțr? ḥr(.j)? nšni=f r ph.t.tj?=[f] ... m hạ.yt
...] der oberste (?) Gott (?), er wütet aus aller Kraft [...] mit einem Regensturm.

Es ist nicht klar, ob nun Amun(-Re) oder wer auch immer hier als höchster Gott angesehen wird, aber man kann sicherlich leicht nachvollziehen, dass Menschen hinter dem bedrohlichen Grollen, Regen, und Wind göttlichen Zorn vermuten und sich vielleicht auch Gedanken darüber machen, wie die Gottheit besänftigt werden kann.

Das Gegenteil davon wäre, sich diesen göttlichen Zorn zu Nutze zu machen. Darauf deutet die Geschichte des Wenamun aus der dritten Zwischenzeit (ca. 1076-943 v. Chr.) hin. Der Protagonist, der im Auftrag der Pharaonen Bauholz für die Götterbarke des Amun in Byblos holen soll, sagt in einer Szene zum Lokalfürsten (P. Moskau inv. 120, Kol. 2, Z. 13 f):

jw=j (r) ‘š sg3-pw r p3 jr-b3-r-n3.w j:jri t3 p.t wn jw n3 ht.w d(j) ḩ3 (hir) sp.t (n) p3 yw-m ${ }^{\circ}$
(Wenn/Sobald) ich laut zum Libanon rufe, werden die Stämme, kaum dass sich der Himmel geöffnet hat, hier an das Ufer des Meeres geworfen (d. h.: sobald ich ein Gebet in Richtung des sakralen Bezirks, den die Berge des Libanon bilden, ausspreche, wird der Wettergott Baal (eventuell mit seinen Blitzen) dafür sorgen, dass die Bäume gefällt werden).

Auch in Kol. 2, Z. 19 preist Wenamun die ägyptischen Götter als Herren über das Wetter:

$m=k j$ j:jri jmn ḩrw $m$ t3 p(.t) jw di=f swth m-rk=f
Siehe, Amun donnert am Himmel, (aber erst) nachdem er Seth neben sich gesetzt hat.

Seth und Baal werden hier als donnernde Götter beschrieben, die sogar Bäume entwurzeln können. Wenamun denkt dabei natürlich an das Bauholz, das er besorgen soll. Doch in der Geschichte bleibt er erfolglos, seine Dienstreise ist ein einziges Fiasko.

Erfolgreicher in der Nutzbarmachung von Niederschlägen ist der ägyptische Magier Hor, Sohn des Panesche, in der römerzeitlichen Überlieferung des Zweiten Setna-Romans, der sich ein Duell mit einem nubischen Magier liefert: In der 
Thronhalle des Pharao lässt der Nubier ein Feuer entstehen, das Hor, Sohn des Panesche, löschen kann (2. Setna Kol. 6, Z. 12-15):

ir p3 3te $n$ igš w'sp-n-ḥjq-(n-)sh tw=f pr t3 ste3.t n p3 wrh=f ‘s pr-‘3 irm n3 ḥt.w n kmj sgp ' 3 iw=w dd gtgte r.ḥr $=\mathrm{n}$ p3 ḥr-tb ḥr s3 p3-nše ir ḥr s3 p3-nše $w^{6}$ hp-n-sh tw=f ir t3 p.t w' haw šm $r$ rj.t-ḥrj.t $n$ t3 ste3.t 'hme $=s$ n t3j ḥtj.t
Der Schamane von Nubien vollbrachte ein Zauberkunststück. Er ließ in der Halle das Feuer hervorkommen. Der Pharao und die Fürsten von Ägypten schrien laut auf, indem sie sagten: „Eile zu uns, Magier Hor, Sohn des Panesche!“ Hor, Sohn des Panesche, machte eine (Zauber-)Formel. Er ließ den Himmel einen oberägyptischen Regen (d.h. eine Sturzflut) über das Feuer ergießen (wörtl. machen). Es verlosch augenblicklich ${ }^{67}$.

Interessant ist, dass der intensive rettende magische Regenschauer als „oberägyptisch“ beschrieben wird, da doch das Gros des Niederschlags in Ägypten an den Küstenregionen fällt. Doch sturzbachartige Regenfälle kamen in der Region um Theben vor und blieben wegen ihrer Seltenheit vermutlich noch mehr in Erinnerung ${ }^{68}$.

Nicht jede Textstelle über Regen ist positiv konnotiert oder nennt einen Gott als Urheber, wie eine Passage aus den Late Egyptian Miscellanies belegt (P. Anastasi 2 = P. BM EA inv. 10243, Kol. 7, Z. 6 f; Parallele P. Sallier 1 = P. BM EA inv. 10185 recto, Kol. 7, Z. 6 f):

p3 ḥm-<ntrr > hạ' m 'ḥw.tj p3 w'b ḥr jri(.t) hn(.t) j:jri=f nw [j]w wn.w 3.t $r$ thb.w $=$ f $\mathrm{m}<$ p3 $>$ jtwr bn sw hr stni $\{\mathrm{r}\}$ pr.t r šmw t3 p.t m țw ḥr mw
Der Pro $<$ phet $>$ steht als Feldarbeiter bereit, der Wab-Priester vollzieht den (Gottes)dienst. Er verbringt die Zeit damit, weil es drei (Dienste) gibt, sich in den Fluß einzutauchen (, um sich $\mathrm{zu}$ reinigen). Er unterscheidet nicht zwischen Winter und Sommer, (auch nicht, ob) der Himmel aus Wind und Wasser (d.h. Regen) besteht (d.h. er

67 Der ägyptische Ausdruck für „oberägyptischen Regen“ ist ḥw šm‘.

68 Vgl. Dorn/Müller 2006. 
muß sich reinigen, egal, was für ein Wetter es ist).

Diese Berufe-Charakteristik erfüllt den Zweck, allein den Schreiberberuf als erstrebenswerte Profession darzustellen. So kann man nachvollziehen, dass die Priesterdienste und die Reinigung als negative Tätigkeiten beschrieben werden, die auch bei Regenwetter vollzogen werden müssen.

\subsection{Regenomina}

Ein bekannteres Omen ist der Tier- oder Blutregen. Dieses Phänomen kann in Teilen naturwissenschaftlich erklärt werden, doch geht es uns hier um das religiöse Phänomen der Omina. Bekannt aus den biblischen Plagen im Buch Exodus 8,2, gibt es dafür auch einen Beleg in einem demotischen Omenbuch der ptolemäischen Zeit, wonach Frösche vom Himmel fallen:

„Wenn die Stimme des Seth spricht (wörtlich: geschieht) und der Himmel Frösche regnet, dann ..." ${ }^{69}$. Aber auch weniger exotische Unwetter wie Regen oder Wind sind verzeichnet. Jedem Omen ist eine Erläuterung beigefügt, wie das jeweilige Wetterphänomen zu deuten ist. Der Bearbeiter Philippe Collombert ist sich sicher, dass dieser - wie auch weitere ägyptische Omenkataloge - durch Praktiken aus dem Vorderen Orient beeinflusst worden sind ${ }^{70}$. Hinter solchen Omina sind göttliche Botschaften zu verstehen, die Menschen mit Hilfe von Nachschlagewerken und mantischen Spezialisten dahingehend $\mathrm{zu}$ deuten versuchen, was sie für ihr individuelles Schicksal oder auch das Ergehen des ganzen Landes aussagen können. Blitz- und Donner als göttliche Stimme spielen eine wichtige Rolle in Omenbüchern ${ }^{71}$.

Im Zweiten Setna-Roman ist es Setnas Gegner, der zu Wettermagie fähig ist: er färbt den Himmel blutrot und lässt Speisen und Wasser blutig sein ${ }^{72}$. In den literarischen Texten sind es immer hochstehende Personen wie Königssöhne, hochrangige Priester oder Wunderkinder, die zu solchen Taten fähig sind - meist am Hofe des Pharaos in der Residenz. Damit wird der Rezipientenschaft gezeigt, dass es sich um herausragende, einmalige Aktionen handelt, zu denen nur diese Protagonisten in der Lage sind.

69 Omina brontoscopiques, Kol. x+1, Z. x+11; vgl. Collombert 2014, 15-26.

70 Collombert 2014, 24.

71 Vernus, 1981; Roccati 1984; Gehlken 2008; MacIntosh Turfa 2012.

72 2. Setna, Kol. 6, Z. 4 f und Kol. 6, Z. 24 f. 
Ich hoffe, dass ich damit exemplarisch zeigen konnte, dass Regen und damit verbundene Wettererscheinungen wie Blitz und Donner für die Fragestellung relevant sein können: Regen bringt fruchtbare Fülle, aber kann auch etwas sein, das eine andere Handlung begleitet, hervorhebt oder verdeckt.

Nach dem Regen kommt die Sonne wieder heraus - personifiziert durch den Gott Re. Das Gesalbt-Werden durch die Strahlen der Sonnenscheibe ist eine Wunschformel im Briefformular und steht im Kontext, dass der Sonnengott die Bitten des Adressaten erhören soll ${ }^{73}$. Er ist mit seinen Strahlen dafür verantwortlich, dass die Menschen sehen können ${ }^{74}$ oder stark werden ${ }^{75}$. In schlimmen Zeiten ist der Sonnengott schwach und scheint nur so hell wie der Mond ${ }^{76}$.

\section{Zusammenfassung und Exkurs zu den magischen Papyri}

Die Ausführungen haben hoffentlich gezeigt, dass die alten Ägypter eine klare Vorstellung davon hatten, dass Diesseits und Jenseits getrennte Sphären waren. Himmel und Erde lassen sich leicht voneinander scheiden; in den Himmel wollen die Toten aufsteigen, um bei den Göttern zu sein ${ }^{77}$. Der Jenseitsbegriff umfasst aber nicht nur den Himmel, sondern auch die Unterwelt, weil nach dem zyklischen Zeit- und Weltverständnis der Ägypter dahinter die Tages- und Nachtfahrt der Sonne steht, die durch Tageshimmel und Nachthimmel führt - oberirdisch

73 Wermai, Kol. 1, Z. 6.

74 Loyalistische Lehre, Stele Kairo CG 20538 des Sehetep-ib-Re, § 2, 5-3, 4, Z. 2, c, 11-13; Parallelen P. Rifeh = P. London UC inv. 32781, § 1, 1-2, Z. 1-3, § 2, 5-3, 4, Z. 5-8 und O. DeM 1235, $\S 2,5-3,4$, Z. 4-7 (innerhalb einer Passage, in der der König gepriesen und mit dem Sonnengott verglichen wird).

75 Satirische Streitschrift, in $\S 38$ f von O. Turin CGT 57313 = Suppl. 6618, O. DeM 1616 und P. Anastasi 1 = P. BM EA inv. 10247 .

76 Neferti, Z. $53 \mathrm{f}$.

77 Siehe auch den Auswertungsbeitrag von Naether und Zomer, Mittel des Transfers vom Himmel mit der Besprechung der Himmelsleiter im vorliegenden Band. Vgl. Brugsch 1890, 580 und ferner Blok 1928; Kees 1930; Griffiths 1964/1965; Griffiths 1966/1967. Auch Papyrusstängel und Stufenpyramiden sind in diesem Zusammenhang relevant; vgl. Alexanian 2003, 34-38 und zum so genannten zšš-w3d-Ritual zu Ehren der Göttin Hathor Altenmüller 2002 mit Belegen aus den Pyramidentexten (Altes Reich, ca. 2700-2200 v. Chr.), den Sargtexten (Mittleres Reich, ca. 2137-1781 v. Chr.) und im „Dramatischen Ramesseumspapyrus“ (ca. 1980 v. Chr.). 
und unterirdisch. Zu schlimmen Krisenzeiten wie Krieg kann diese Ordnung erschüttert werden und die Menschen müssen fürchten, dass der Himmel auf sie herniederstürzen kann.

Der Himmel ist ganz klar die göttliche Sphäre. Von dort kommen die Götter, wenn sie die Menschen besuchen, von dort senden sie als Hylemelemente Entitäten wie Dämonen, Krankheiten oder materielle Geschenke.

Durch magische Praktiken können sich entsprechend ausgebildete Menschen dies zu Nutze machen. Dies zeigt die Beschäftigung mit den griechischen und demotischen magischen Papyri (Papyri Graecae/Demoticae Magicae; PGM und PDM $)^{78}$. Es wäre müßig, alle Belege zu Invokationen der Götter und Dämonen im Himmel aufzulisten - ganz oft werden bei Helios, Horus, Iao usw. auf deren Aufenthaltsort im Himmel abgehoben. Dieser wird oft differenziert nach den vier Himmelsrichtungen, den sieben Himmeln, Stufen zwischen Himmel und Erde oder dem Himmel als ein Teil des Kosmos neben Erde, Wasser und Bergen, den der jeweilige Gott beherrscht. Außerdem wird oft erwähnt, dass der magische Praktiker bei Invokationen zum Himmel blicken oder Rituale unter freiem Himmel vollziehen soll. Für die Fragestellung relevant sind folgende Zaubersprüche, die ich hier kurz in Tabellenform notiere. Sie sind allesamt griechisch verfasst, die letzten beiden fasste Preisendanz noch unter „Christliches“.

Tab. 1: Übersicht zu den Zaubersprüchen

\begin{tabular}{ll}
\hline Textstelle & Inhalt \\
\hline PGM 1, 42-195 & $\begin{array}{l}\text { Falke bringt Stein für Amulett zu magischem Praktiker, Engel } \\
\text { kommt als Stern vom Himmel herab und gibt Orakel }\end{array}$ \\
PGM 2, 80-85 & $\begin{array}{l}\text { Erwähnung, dass leios/Paian vom Himmel kommt } \\
\text { Erwähnung, dass Pythischer Paian vom Himmel kommt } \\
\text { PGM 3, 259f }\end{array}$ \\
PGM 4, 1015-1020 & $\begin{array}{l}\text { Engerufener Gott (Horus/lao etc.) ist im Himmel geboren } \\
\text { PGM 4, 1115-1160 }\end{array}$ \\
(Himmelsstütze) \\
PGM 4, 1610
\end{tabular}

78 Preisendanz 1973/1974²; Betz 1992². 


\begin{tabular}{ll}
\hline Textstelle & Inhalt \\
\hline PGM 12, 14-95 & Traumbitte: Gott soll aus Himmel kommen \\
PGM 12, 96-106 & Traumbitte: Gott soll aus Himmel kommen \\
PGM 12, 107-121 & $\begin{array}{l}\text { Traumbitte: Gott soll aus Himmel kommen } \\
\text { PGM 12, 201-296 }\end{array}$ \\
PGM 36, 295-311 & $\begin{array}{l}\text { Engel kommen vom Himmel nach Sodom und Gomorrha (in histo- } \\
\text { riola) }\end{array}$ \\
PGM 15 a („Christliches“) & $\begin{array}{l}\text { Licht kommt zur Erde durch geöffnete Schleusen des Himmels } \\
\text { PGM 15 b („Christliches“) }\end{array}$ \\
\hline
\end{tabular}

Auffällig ist dabei, dass auf Demotisch oder Hieratisch verfasste Texte nicht vorkommen. Jedoch sind die Religionen der Verfasser der magischen Papyri vielfältig, wie sich auch in diesen Beispielen zeigt: ägyptische, griechisch-römische, jüdische und christliche Entitäten lassen sich finden. Um den Menschen Träume oder andere Offenbarungen zu senden, steigen die Götter vom Himmel auf die Erde herab. Die Schilderungen zur Schöpfung des Kosmos sind gut mit denen in den ägyptischen literarischen Texten in Einklang zu bringen.

\section{Literaturverzeichnis}

Alexanian, N., 2003, Himmelstreppen und Himmelsaufstieg. Zur Interpretation von Ritualen auf Grabdächern im Alten Reich, in: Guksch, H./Hofmann, E./Bommas, M. (Hg.), Grab und Totenkult im Alten Ägypten, München, 27-40.

Altenmüller, H., 2002, Der Himmelsaufstieg des Grabherrn. Zu den Szenen des zšš w3d in den Gräbern des Alten Reiches, in: Studien zur Altägyptischen Kultur 30, 1-42.

Ayali-Darshan, N., 2010, „The Bride of the Sea“: The Traditions about Astarte and Yamm in the Ancient Near East, in: Horowitz, W./Gabbay, U./Vukosavović, F. (Hg.), A Woman of Valor. Jerusalem Ancient Near Eastern Studies in Honor of Joan Goodnick Westenholz, Biblioteca del Próximo Oriente Antiguo 8, Madrid, 19-33.

Baud, M., 2010, Taharqa, l'offrande au faucon Hémen et le miracle de la pluie, in: Baud, M./Sackho-Autissier, A./Labbé-Toutée, S. (Hg.), Méroé. Un empire sur le Nil, Paris, 173 f.

Betz, H. D., 1992, The Greek Magical Papyri in Translation. Including the Demotic Spells,

2. Aufl., Chicago/London (1. Aufl. 1986).

Bickel, S., 2005, Creative and Destructive Waters, in: Amenta, A./Luiselli, M. M./Sordi, M. N. (Hg.), L'acqua nell'antico Egitto: vita, rigenerazione, incantesimo, medicamento, Proceedings of the First International Conference for Young Egyptologists, Italy, Chianciano Terme, October 15-18, 2003, Rom, 191-200.

Blok, H. P., 1928, Zur altägyptischen Vorstellung von der Himmelsleiter, in: Acta Orientalia 6, 257-269.

Bonnet, H., 1952, Reallexikon der ägyptischen Religionsgeschichte, Berlin. 
Borghouts, J. F., 1984, The First Hittite Marriage Record: Seth and the Climate, in: Institut d’Égyptologie, Université Paul Valéry (Hg.), Mélanges Adolphe Gutbub, Orientalia Monspeliensia 2, Montpellier, 13-16.

Braun, N. S., 2013, Pharao und Priester - Sakrale Affirmation von Herrschaft durch Kultvollzug. Das Tägliche Kultbildritual im Neuen Reich und der Dritten Zwischenzeit, Philippika 23, Wiesbaden.

Brugsch, H., 1890, Religion und Mythologie der alten Aegypter. Nach den Denkmälern. Aufstieg des Toten auf der Himmelsleiter, Leipzig.

Cameron, A., 2004, Greek Mythography in the Roman World, American Classical Studies 48, Oxford u. a.

Collombert, P., 2014, Omina brontoscopiques et pluies de grenouilles, in: Depauw, M./Broux, Y. (Hg.), Acts of the Tenth International Congress of Demotic Studies, Leuven, 26-30 August 2008, Orientalia Lovaniensia Analecta 231, Leuven, 15-26.

Collombert, P./Coulon, L., 2000, Les dieux contre la mer. Le début du „papyrus d'Astarté“ (pBN 202), in: Bulletin de l'Institut Français d'Archéologie Orientale 100, 193-242.

Comelli, D. et al., 2016, The Meteoritic Origin of Tutankhamun's Iron Dagger Blade, in: Meteoritics \& Planetary Science 51, 1301-1309, https://onlinelibrary.wiley.com/doi/full/10.1111 /maps.12664 (Abruf 13.5.2021)

Dieleman, J., 1998, Fear of Women? Representations of Women in Demotic Wisdom Texts, in: Studien zur Altägyptischen Kultur 25, 7-46.

Dorman, P. F., 1999, Creation on the Potter's Wheel at the Eastern Horizon of Heaven, in: Teeter, E./Larson, J. A. (Hg.), Gold of Praise. Studies on Ancient Egypt in Honor of Edward F. Wente, Studies in Ancient Oriental Civilization 58, Chicago, 83-99.

Dorn, A., 2016, The Hydrology of the Valley of the Kings. Weather, Rainfall, Drainage Patterns, and Food Protection in Antiquity, in: Wilkinson, R. H./Weeks, K. R. (Hg.), The Oxford Handbook of the Valley of the Kings, Oxford, 30-38.

Dorn, A./Müller, M., 2006, Regenfälle in Theben-West, in: Zeitschrift für Ägyptische Sprache und Altertumskunde 133, 90-93.

Enmarch, R., 2008, A World Upturned: Commentary on and Analysis of The Dialogue of Ipuwer and the Lord of All, Oxford $u$. a.

Erichsen, W., 1954, Demotisches Glossar. Kopenhagen.

Fischer-Elfert, H.-W., 2014, Ein Spruch gegen den Bösen Blick in Meroë: Anmerkungen zur Bronzeschale Boston MFA 24.900 aus Grab S 155 der Süd-Nekropole, in: Orientalia. Nova Series 83, 31-49.

Gehlken, E., 2008, Die Adad-Tafeln der Omenserie Enūma Anu Enlil. Teil 2: Die ersten beiden Donnertafeln (EAE 42 und EAE 43), in: Zeitschrift für Orientarchäologie 1, 256-314.

Goebs, K., 2002, A Functional Approach to Egyptian Myth and Mythemes, in: Journal of Ancient Near Eastern Religions 2, 27-59.

Graves-Brown, C., 2007, Flint and the Northern Sky, in: Schneider, T./Szpakowska, K. (Hg.), Egyptian Stories. A British Egyptological Tribute to Alan B. Lloyd on the Occasion of His Retirement, Alter Orient und Altes Testament 347, Münster, 111-135.

Griffiths, J. G., 1964/1965, The Celestial Ladder and the Gate of Heaven (Genesis XXVIII. 12 and 17), in: The Expository Times 76, $229 \mathrm{f}$.

Griffiths, J. G., 1966/1967, The Celestial Ladder and the Gate of Heaven in Egyptian Ritual, in: The Expository Times 78, $54 \mathrm{f}$.

Grimm, J./Grimm, W., 2002, Kinder- und Hausmärchen. Vollständige Ausgabe, 19. Aufl., Düsseldorf/Zürich. 
Gros, E., 1870, Histoire Romaine de Dion Cassius. Tome dixième. Traduite en Français avec des notes critiques, hist. et le texte en regard, par E. Gros, Paris.

Guey, J., 1948, Encore la Pluie miraculeuse. Mage et Dieu, Revue de Philologie, de Littérature et d'Histoire Anciennes, 3e série 22, 16-62.

Guilhou, N., 2010, Myth of the Heavenly Cow, in: Dieleman, J./Wendrich, W. (Hg.), UCLA Encyclopedia of Egyptology, Los Angeles, http://digital2.library.ucla.edu/viewltem.do?ark $=21198 / \mathrm{zz} 002311 \mathrm{pm}$ (Abruf 13.5.2021)

Hawes, G., 2014, Rationalizing Myth in Antiquity. Oxford/New York.

Helck, W., 1995, Eisen, in: Helck, W./Otto, E. (Hg.), Lexikon der Ägyptologie, Band 1, Wiesbaden, Sp. $1209 \mathrm{f}$.

Hoffmann, F., 1996, „Der Kampf um den Panzer des Inaros“. Studien zum P. Krall und seiner Stellung innerhalb des Inaros-Petubastis-Zyklus, Mitteilungen aus der Papyrussammlung der Österreichischen Nationalbibliothek (Papyrus Erzherzog Rainer). Neue Serie 26, Wien.

Hoffmann, F./Quack, J. F., 2007, Anthologie der demotischen Literatur, Einführungen und Quellentexte zur Ägyptologie 4, Berlin u. a.

Hornung, E., 1982, Der ägyptische Mythos von der Himmelskuh. Eine Ätiologie des Unvollkommenen, Orbis Biblicus et Orientalis 46, Freiburg/Göttingen.

Jaggi, R., 2008, Das Wasser, das vom Himmel kommt, in: Kemet. Die Zeitschrift für Ägyptenfreunde 17 (4), 73-75.

Johnson, D./Tyldesley J., 2014, Iron from the Sky, in: Geoscientist 24 (3), 10-15.

Johnson, D./Tyldesley, J., 2016, Iron from the Sky. The Role of Meteorite Iron in the Development of Iron Working Techniques in Ancient Egypt, in: Price, C. et al. (Hg.), Mummies, Magic and Medicine in Ancient Egypt. Multidisciplinary Essays for Rosalie David, Manchester, 408-423.

Johnson, D./Tyldesley, J./Lowe, T./Withers, P. J./Grady, M. M., 2013, Analysis of a Prehistoric Egyptian Iron Bead with Implications for the Use and Perception of Meteorite Iron in Ancient Egypt, in: Meteoritics \& Planetary Science 48 (6), 997-1006, https://onlinelibrary. wiley.com/doi/full/10.1111/maps.12120 (Abruf 13.5.2021)

Jørgensen, J. K. B., 2013, Egyptian Mythological Manuals. Mythological Structures and Interpretative Techniques in the Tebtunis Mythological Manual, the Manual of the Delta and Related Texts, Dissertation Kopenhagen.

Jung, C., 2007, Rain in Ancient Egypt: A Linguistic Approach, in: Bollig, M. et al. (Hg.), Aridity, Change and Conflict in Africa. Proceedings of an International ACACIA Conference Held at Königswinter, Germany, October 1-3, 2003, Colloquium Africanum 2, Köln, 331-344.

Kees, H., 1930, Die Himmelsreise im ägyptischen Totenglauben, in: Saxl, F. (Hg.), Über die Vorstellungen von der Himmelsreise der Seele, Vorträge der Bibliothek Warburg 8 (Vorträge 1928/1929), Leipzig/Berlin, 1-20.

Kovács, P., 2008, Das Regenwunder Marc Aurels, in: Acta Antiqua Academiae Scientiarum Hungaricae 48, 387-404.

Kurth, D., 1975, Den Himmel stützen.Die „tw3 pt“-Szenen in den ägyptischen Tempeln der griechisch-römischen Epoche, Rites Égyptiens 2, Brüssel.

Leblanc, C., 1995, Thèbes et les pluies torrentielles. A propos de mw m pt, in: Memnonia 6, 197-214.

Lichtheim, M., 1983, Late Egyptian Wisdom Literature in the International Context. A Study of Demotic Instructions, Orbis Biblicus et Orientalis 52, Freiburg/Göttingen.

MacIntosh Turfa, J., 2012, Divining the Etruscan World. The Brontoscopic Calendar and Religious Practice, Cambridge u. a. 
Naether, F., 2010, Die Sortes Astrampsychi. Problemlösungsstrategien durch Orakel im römischen Ägypten, Orientalische Religionen in der Antike 3, Tübingen.

Pongratz-Leisten, B., 2015, Religion and Ideology in Assyria, Studies in Ancient Near Eastern Records 6, Boston/Berlin.

Posener, G., 1951, À propos de la „Pluie Miraculeuse“, in: Revue de Philologie, de Littérature et d'Histoire Anciennes, 3e série 25, 162-168.

Preisendanz, K., 1973/1974, Papyri Graecae Magicae. Die Griechischen Zauberpapyri. Zweite Auflage mit Ergänzungen von K. Preisendanz, durchgesehen und herausgegeben von A. Henrichs, Stuttgart (1. Aufl. Leipzig 1928/1931).

Quack, J. F., 1994, Die Lehren des Ani, Orbis Biblicus et Orientalis 141, Freiburg/Göttingen.

Quack, J. F., 2012, Danaergeschenk des Nil? Zu viel oder zu wenig Wasser im Alten Ägypten, in: Berlejung, A. (Hg.), Disaster and Relief Management - Katastrophen und ihre Bewältigung, Forschungen zum Alten Testament 81, Tübingen, 333-381.

Ritner, R. K., 1993, The Mechanics of Ancient Egyptian Magical Practice, Studies in Ancient Oriental Civilizations 54, Chicago.

Roccati, A., 1984, Lessico meteorologico, in: Junge, F. (Hg.), Studien zu Sprache und Religion Ägyptens. Zu Ehren von Wolfhart Westendorf, überreicht von seinen Freunden und Schülern, Band I, Göttingen, 343-354.

Sauneron, S., 1952, Un thème littéraire de l'antiquité classique: le Nil et la pluie, Bulletin de l'Institut Français d'Archéologie Orientale 51, 41-48.

Schäfer, H., 1931, Isis Regengöttin?, in: Zeitschrift für Ägyptische Sprache und Altertumskunde 66, 139.

Smith, H. S./Tait, W. J., 1983, Saqqâra Demotic Papyri I, Texts from Excavations 7, London.

te Velde, H., 1977, The Theme of the Separation of Heaven and Earth in Egyptian Mythology, in: Kákosy, L./Gaál, E. (Hg.), Studia Aegyptiaca 3, Budapest, 161-170.

Theis, C., 2014, Magie und Raum. Der magische Schutz ausgewählter Räume im Alten Ägypten nebst einem Vergleich zum angrenzenden Kulturbereichen, Orientalische Religionen in der Antike 13, Tübingen.

Thissen, H.-J., 2004, „Wer lebt, dessen Kraut blüht!“. Ein Beitrag zu demotischer Intertextualität, in: Hoffmann, F./Thissen, H.-J. (Hg.), Res severa verum gaudium. Festschrift für KarlTheodor Zauzich zum 65. Geburtstag am 8. Juni 2004, Studia Demotica 6, Leuven/Paris/Sterling, 583-594.

Tóth, I., 1976, Marcus Aurelius' Miracle of the Rain and the Egyptian Cults in the Danube Region, in: Kákosy, L./Gaál, E. (Hg.), Studia Aegyptiaca 2, Budapest, 101-113.

Vernus, P., 1981, Omina calendériques et comptabilité d'offrandes sur une tablette hiératique de la XVIIle dynastie, in: Revue d'Égyptologie 33, 89-124.

Vittmann, G., 1998, Der demotische Papyrus Rylands 9, Ägypten und Altes Testament 38, Wiesbaden.

Weeks, N. K., 2015, Myth and Ritual: An Empirical Approach, in: Journal of Ancient Near Eastern Religions 15, 92-111.

Weyersberg, M., 1961, Das Motiv der „Himmelsstütze“ in der altägyptischen Kosmologie, in: Zeitschrift für Ethnologie 86, 113-140.

Wilke, C., 1931, Ein Regenzauber in den Pyramidentexten?, in: Zeitschrift für Ägyptische Sprache und Altertumskunde 67, $127 \mathrm{f}$.

Yalçın, Ü., 2005, Zum Eisen der Hethiter, in: Yalçın, Ü./Pulak, C./Slotta, R. (Hg.), Das Schiff von Uluburun - Welthandel vor 3000 Jahren, Katalog der Ausstellung des Deutschen Bergbau- 
Museums Bochum vom 15. Juli 2005 bis 16. Juli 2006, Veröffentlichungen aus dem Deutschen Bergbau-Museum Bochum 138, Bochum, 493-502.

Zgoll, C., 2019, Tractatus mythologicus. Theorie und Methodik zur Erforschung von Mythen als Grundlegung einer allgemeinen, transmedialen und komparatistischen Stoffwissenschaft, Mythological Studies 1 (https://doi.org/10.1515/9783110541588), Berlin/Boston. 